\title{
The Medium Chain Triglyceride diet and intractable epilepsy
}

\author{
M A SILLS, W I FORSYTHE, D HAIDUKEWYCH, A MACDONALD, AND M ROBINSON \\ General Infirmary and St James's University Hospital, Leeds, and Epilepsy Centre of Michigan, Detroit, \\ United States of America
}

SUMMARY Fifty children with drug resistant epilepsy were treated with the Medium Chain Triglyceride (MCT) Emulsion diet. Eight achieved complete control of seizures (four without anticonvulsant drugs), and with the addition of anticonvulsants four had seizures reduced in frequency by $90 \%$ and 10 by $50-90 \%$. The best results were obtained with astatic myoclonic and absence seizures, but control of seizures was improved in four children with tonic-clonic and three with complex partial seizures. Food given at the same time as MCT helped to reduce side effects, and an extra dose of MCT before bedtime improved control of nocturnal seizures.

Sixty years ago Geyelin found that many patients with epilepsy remained free of seizures while fasting and for several months after return to a normal diet. ${ }^{1}$ Wilder in the same year suggested that the ketonaemia induced by fasting was responsible for this effect and proposed that a 'ketogenic diet', high in fat and low in carbohydrate, should be used in the treatment of epilepsy. ${ }^{2}$ This was undertaken in children by Peterman ${ }^{3}$ and Helmholtz, ${ }^{4}$ and the best results seemed to occur in the younger age groups. Livingston reported over a period of 41 years that he had controlled myoclonic epilepsy in $54 \%$ of 975 cases using the ketogenic diet. ${ }^{5}$ In spite of these good results, however, the traditional ketogenic diet ( $87 \%$ of dietary energy being provided by fat) has been limited by its unpalatability and nutritional inadequacy in young children. It is also difficult to prepare and has not gained wide acceptance.

Recently, there has been a resurgence of interest in ketogenic diets as many children with seizuresfor example, tonic-clonic and astatic myoclonic-are not controlled by anticonvulsants used singly or in combination. ${ }^{6}$ After reports that medium chain triglyceride (MCT) was more ketogenic than fat Huttenlocher et al introduced MCT as an alternative to fat in their ketogenic diet. ${ }^{7}$ They showed that a ketogenic diet using MCT to provide $60 \%$ of dietary energy was at least as effective as a 3:1 ketogenic diet ( $75 \%$ of energy provided by fat) in producing ketosis and controlling seizures. As less carbohydrate restriction was involved such a diet became easier to prepare, was more palatable, and was less lipogenic. Schwartz et al reported success with both the traditional ketogenic diet and the MCT Oil diet and investigated some of its biochemical effects. ${ }^{8}$ More recently, because of the increasing expense of a traditional ketogenic diet and the unpalatability of MCT Oil Schwartz et al have introduced a combined? diet, named the John Radcliffe II diet, which contains smaller volumes of MCT Oil and includes some saturated fat in the form of cream and butter, etc. ${ }^{9}$ Some success with both continued seizure control and improved acceptability has been reported with this innovation. In both these series of Schwartz et $a^{8}{ }^{8}$ the MCT Oil was incorporated into the diet as an emulsion with skimmed milk.

Several theories have been suggested about the possible mode of action of these diets.

(i) Millichap et al reported lowering of the serum $\mathrm{pH}$ with the ketogenic diet, ${ }^{10}$ but this was not observed by Huttenlocher $e t$ al with the MCT diet, ${ }^{7}$ and no $\mathrm{pH}$ reduction was seen in the studies of de Vivo et al of long term ketogenic diets. ${ }^{11}$

(ii) Millichap et al suggested that negative sodium and potassium balance was a factor, ${ }^{10}$ but subsequent studies found no evidence of this. ${ }^{78}$

(iii) Dekaban suggested that hyperlipidaemia might have an anticonvulsant effect, ${ }^{12}$ but although raised lipids have been found with the ketogenic diet, this was not observed with the MCT diet, ${ }^{7}$ and also no changes in cholesterol or high density lipoprotein proportions have been observed with the diet. $^{8}$

(iv) Severe hypoglycaemia has been reported by 
Livingston, ${ }^{13}$ but this was not observed in other studies. $^{78}$

(v) Reduction in serum alanine concentrations have been reported with the ketogenic but not the MCT diet. ${ }^{8}$

(vi) Huttenlocher reported rises of plasma concentrations of the ketone bodies $\beta$-hydroxybutyrate and acetoacetate, and his elegant studies suggested the effectiveness of the diet might be related to blood concentrations of these substances. ${ }^{14}$ It is interesting to note that during starvation brain metabolism may alter so that ketone bodies can be utilised as an energy source instead of glucose ${ }^{15}$ and that acetoacetate has been shown to have an anticonvulsant effect in experimental animals. ${ }^{16}$ Huttenlocher showed that ketone concentrations in plasma rose equally with the ketogenic or MCT diets and that this rise was less in older children, in whom the diet seemed less effective. ${ }^{14}$

(vii) Huttenlocher et al suggested the possibility of a direct effect of the fatty acids contained in MCT on cerebral excitability. ${ }^{7}$

We initially attempted to treat 25 children with intractable seizures using the MCT Oil diet but were unable to achieve reasonable palatability, and most patients found it unacceptable. One boy aged 14 with drug resistant absence seizures did persevere with the diet and achieved an impressive improvement in seizure control. After this success we wondered if we might be able to increase the acceptability of the diet by using a proprietary MCT Emulsion as the mechanism of action of this diet would be essentially the same as with the MCT Oil diet, whose efficacy has been well documented. 7814 is

\section{Patients and methods}

In this study MCT Emulsion (Liquigen Scientific Hospital Supplies Ltd, Liverpool) was given. Flavouring was added-namely, Nesquik or synthetic butterscotch-to make the MCT Emulsion more palatable, but several children were able to take it without flavouring. Children admitted to the study were suffering from either astatic myoclonic absence, generalised tonic-clonic, or complex partial seizures, which had failed to respond to the appropriate anticonvulsant given as a single agent. Only children who we considered had a good chance of achieving reasonable function at normal or special school were included in the study.

All children were admitted to hospital and anticonvulsants were withdrawn and were reintroduced only if clinically indicated. Daily energy requirement was assessed on an individual basis by the hospital dietitian. An initial starvation period of one to four days was used to induce ketosis, and then the amount of MCT was slowly increased until $60 \%$ of the dietary energy requirement was supplied by MCT. All children received daily supplements of vitamins, iron, and calcium (usually Ketovite syrup $5 \mathrm{ml}$ daily, Ketovite/tablet three times a day, Sytron $5 \mathrm{ml}$ three times a day, and Calcium Sandoz one tablet daily). The average time to build up to full diet was 18 days. Once established on full diet children were allowed home and were reviewed after two weeks. If the seizures were not in remission an anticonvulsant was added. Children continued on the diet for at least two years if well tolerated. Where difficulties with compliance occurred some children were admitted to a residential hospital school. Seizure frequency before beginning the diet and once established on full diet was assessed from records of seizures kept in hospital by medical and nursing staff and at home by parents. We graded control according to percentage reduction of seizure frequency after stabilisation on the diet: complete control represented $100 \%$, excellent control $90-100 \%$, good control $50-90 \%$, and poor control $<50 \%$ reduction of seizure frequency.

\section{Results}

Fifty children with seizures resistant to appropriate anticonvulsants have been treated with MCT Emulsion, 18 with astatic myoclonic, 17 with generalised tonic-clonic, six with absence, one with absence and tonic-clonic, and eight with complex partial seizures.

Twenty four children tolerated full diet-namely, $60 \%$ MCT -18 tolerated $50-58 \%$, two tolerated $45 \%$, and six were unable to tolerate the diet. Of the 44 children who tolerated the diet, eight achieved complete control of seizures (four without anticonvulsants), four achieved excellent control, and 10 achieved good control. Of the remaining 22 children, 13 had poor control and nine showed no improvement. Of the 22 children with good to complete control, however, three whose control of seizures was excellent refused to continue the diet. Fifteen children have been on the diet for two years or more and seven for more than a year. Sixteen children remain on the diet, eight with astatic myoclonic, three with absence, three with generalised tonic-clonic, and two with complex partial seizures. Four children, two with astatic myoclonic and two with absence seizures, have been off the diet nine months or longer without recurrence of seizures.

Of the 17 children over the age of 10 , one achieved complete, three excellent, seven good, and 
four poor control of seizures and two were unable to tolerate the diet. Success was often associated with a high level of patient motivation. A boy aged 10 opted to try the diet as an alternative to temporal lobotomy and achieved good control, and a girl aged 11 who had developed anorexia nervosa on MCT Oil was determined to try MCT Emulsion and achieved excellent control of her absence seizures but required the addition of an anticonvulsant (ethosuximide) to achieve complete control.

In addition, 11 children had considerable difficulties with compliance because they would not take the diet or their parents would not administer it properly, despite the fact that five of them had achieved good control or better. Admission to a residential hospital school was accepted by nine of them, and eight achieved good to complete control.

The timing of seizures was of interest: eight children whose seizures had been diurnal before treatment with the diet had seizures only at night on full diet. These nocturnal seizures ceased when an extra bolus of MCT was given at 2000 to $2100 \mathrm{~h}$, but it was noted that diarrhoea and abdominal pain occurred if extra MCT was not given with food-for example, biscuits.

\section{Results of different seizure types.}

Astatic myoclonic

All 18 of the children in this group (who were aged 2-8 years, with a mean of 4 years) had been tried on sodium valproate, nitrazepam, and clonazepam without reduction of seizure frequency. The results of treatment on MCT diet alone or with sodium valproate or nitrazepam are shown in the Table.

\section{Absence}

All of the six children with 'pure absence' seizures and the child with absence and generalised tonicclonic seizures (who were aged 5-11 years, with a mean of 8 years) had been tried on sodium valproate, ethosuximide, methsuximide, and acetazolamide without reduction of seizure frequency.
The child with tonic-clonic seizures had also been tried unsuccessfully on carbamazepine and phenytoin. The results of treatment on MCT diet plus sodium valproate or ethosuximide are shown in the Table.

\section{Generalised tonic clonic}

All of the 17 children in this group (who were aged $5-15$ years, with a mean of 10 years) had been tried on carbamazepine, phenytoin, primidone, sodium valproate, and each combined with acetazolamide without reduction of seizure frequency. The results of treatment on MCT diet plus an anticonvulsant are shown in the Table.

\section{Complex partial}

All of the eight children in this group (who were aged 9-14 years, with a mean of 10 years) had been tried on carbamazepine, phenytoin, primidone, sodium valproate, and each combined with acetazolamide without reduction of seizure frequency. The results of treatment on MCT diet plus carbamazepine or phenytoin are shown in the Table.

Side effects. Mild diarrhoea and abdominal pain were common and were usually alleviated by a temporary reduction of MCT dosage or when MCT was taken in small amounts with food. Side effectse led three children, however, who had had excellent? control to give up the diet, two of whom have restarted the diet with excellent control.

In 13 children a subjective improvement in mental awareness was observed by parents and nursing and school staff.

One boy aged 10 with severe astatic myoclonic seizures of three years' duration showed a good initial response to the MCT Emulsion diet but later refused to take it. The diet was given by nasogastric tube, but we omitted to give him vitamins through the tube and four weeks later he became blind. His sight was restored four weeks after starting treatment with vitamins.

Table Control of the different types of seizure on the MCT diet in 50 children with drug resistant epilepsy

\begin{tabular}{|c|c|c|c|c|c|c|}
\hline \multirow[t]{2}{*}{ Type of seizure } & \multirow{2}{*}{$\begin{array}{l}\text { No of } \\
\text { children }\end{array}$} & \multicolumn{5}{|l|}{ Control } \\
\hline & & $\begin{array}{l}\text { Complete } \\
(10(0 \%)\end{array}$ & $\begin{array}{l}\text { Excellent } \\
(90-100 \%)\end{array}$ & $\begin{array}{l}\text { Good } \\
(.50-90 \%)\end{array}$ & $\begin{array}{l}\text { Poor } \\
(50 \%)\end{array}$ & Failed \\
\hline Astatic myoclonic & 18 & 3 & 1 & 5 & 5 & 4 \\
\hline Petit mal & 7 & 3 & 2 & 1 & 1 & - \\
\hline Tonic-clonic & 17 & 1 & 1 & 2 & 7 & 6 \\
\hline Complex partial & 8 & 1 & - & 2 & - & 5 \\
\hline Total & 50 & 8 & 4 & 10 & 13 & 15 \\
\hline
\end{tabular}




\section{Discussion}

These results suggest as previously reported ${ }^{7} 817$ that the MCT diet is worth considering for children with drug resistant seizures and that using a proprietary MCT Emulsion may improve acceptability.

In contrast to previous studies ${ }^{89}$ we withdrew anticonvulsants before introducing the diet. We believe it makes no sense to continue to dispense anticonvulsant treatment that is ineffective, but we insist that withdrawal takes place in hospital. The fact that four children with astatic myoclonic seizures managed better on MCT diet off drugs we think justified this approach and also gives credence to the view that the MCT diet contains 'agents' that have anticonvulsant properties. In addition, we have treated older children with a variety of seizures with some success, and this suggests that the MCT diet should be considered in any child of reasonable intelligence suffering drug resistant seizures rather than reserving it for the younger child as has been suggested. ${ }^{14}$

Side effects remain a problem, and we have found that the probable osmotic effects of the diet may be alleviated by giving MCT in regular small amounts with food.

Bilateral optic neuropathy has been reported in two children aged 5-7 who were maintained on a 4:1 ketogenic diet without vitamin B supplements. ${ }^{19}$ Blindness occurred 10 months and one year, respectively, after introduction of the diet. Vitamin B supplements and thiamine restored vision in six and 11 weeks, respectively. Our own experience emphasises the importance of giving adequate vitamin B supplements while taking MCT Emulsion.

The reduction of nocturnal seizures afforded by extra MCT at night may relate to the paradoxically low morning concentrations of ketones in children on the MCT diet that have been reported previously. ${ }^{818}$ Our biochemical observations, reported elsewhere, shed some light on the reasons for this. ${ }^{20}$

It is difficult to compare the results of treatment of our 50 children with the 54 treated by Schwartz et $a l^{9}$ because different seizure types were treatednamely, infantile spasms and partial seizures-and the duration of treatment was not specified, whereas we have attempted to assess control of seizures over the whole duration of treatment, with 22 out of 44 children having received treatment for at least a year at the time of assessment. In their series of 25 children with 'drop seizures', however, $85 \%$ had $>50 \%$ reduction in seizure frequency, whereas in our series of 18 children with astatic myoclonic seizures $50 \%$ had $>50 \%$ reduction in seizure frequency. They provided no information on the eight children they treated with absence seizures, and it is unclear which of the three diets suited the individual children best.

We realise that much of our data on seizure frequency relies on second hand information, but it is difficult to improve on this without either long periods of admission to hospital or prolonged electrical recordings. More reliable records were obtained when nine children were admitted to a residential hospital school for long periods, where teachers and nurses recorded all seizures and the degree of control consequent on this-that is, four complete control, two excellent control, two good control and one would not comply. Two of the children who obtained complete control for six months and then refused the diet did not relapse for three to six months after the diet was withdrawn.

It is our routine to continue the diet and if necessary anticonvulsants for two years after the last seizure. The anticonvulsant is withdrawn slowly and two months later the diet is stopped.

When we see a child with numerous astatic myoclonic seizures in a padded bed for his own protection, drooling, unable to speak or feed himself, and in two weeks on $48 \%$ MCT running around the ward, remaining free of seizures for two years and now off treatment for a year without seizures, we begin to ask ourselves if MCT Emulsion should be the first choice treatment for this type of seizure.

We thank the nursing and dietetic staff of the paediatric units at the General Infirmary and St James's University Hospital, Leeds, for their unfailing efforts, Mrs $\mathbf{J}$ Crossley and Miss $\mathrm{H}$ Shelton for secretarial help, and the nursing staff and teachers at Wharfedale Children's Hospital for their help with these 'difficult' children.

\section{References}

1 Geyelin HR. Fasting as a method of treating epilepsy. Medical Record 1921;99:1037-9.

2 Wilder RM. The effect of ketonaemia on the cause of epilepsy. Mayo Clinic Bulletin 1921:2:307-14.

${ }^{3}$ Peterman MS. The ketogenic dict in epilepsy. JAMA 1925;84:1979-83.

${ }^{4}$ Helmholz HF. The treatment of epilepsy in childhood: 5 years experience of the ketogenic dict. JAMA 1927;88:2028-32

${ }^{5}$ Livingston $\mathrm{S}$. Ketogenic diet in the treatment of childhood cpilepsy. Dev Med Child Neurol 1977;19:833-4.

${ }^{6}$ Forsythe WI, Sills MA. One drug for childhood grand mal: a medical audit for 3 year remissions. Dev Med Child Neurol 1984;26:742-8.

${ }^{7}$ Huttenlocher PR, Wilborne AJ, Signore JM. Medium-chain triglycerides as a therapy for intractable childhood epilepsy. Neurology 1971;21:1097-103.

* Schwartz RH, Aynsley-Green A, Bower BP. Clinical and metabolic aspects of the ketogenic diets. Research and Clinical Forums 1980;2:63-73.

"Schwartz RH. Eaton J, Aynsley-Green A, Bower BD. Ketogenic diets in the management of childhood epilepsy. In: Rose FC, ed. Research progress in epilepsy. London: Pitman, 1983:326-32. 
10 Millichap JC, Jones JD, Rudis BP. Mechanism of anticonvulsant action of ketogenic diets. Am J Dis Child 1964;107:593-604.

" De Vivo DC, Pagliara AS, Prensky AL. Ketotic hypoglycaemia and the ketogenic diet. Neurology 1973:23:640-9.

12 Dekaban AS. Plasma lipids in epileptic children treated with fat. Arch Neurol 1966;15:177-84.

13 Livingston S. Diagnosis and treatment of childhood myoclonic seizures. Pediatrics 1974:53:542-8.

14 Huttenlocher PR. Ketonaemia and seizures: metabolic and anticonvulsant effects of two ketogenic diets in childhood epilepsy. Pediatr Res 1976;10:536-40.

15 Owen CE, Morgan AP, Kemp HG, Sullivan JM, Herrena MG, Cahil G. Brain metabolism during fasting. J Clin Invest 1967;46:1585-94.

${ }^{16}$ Keith HM. Convulsive disorders in children with reference to treatment with ketogenic diet. Boston: Little Brown, 1963.
17 Gordon N. Medium chain triglycerides in a ketogenic diet. Dev Med Child Neurol 1977;19:535-44.

${ }^{18}$ Clark BJ, House FM. Medium chain triglyceride oil ketogenic diets in the treatment of childhood epilepsy. Journal of Human Nutrition 1978;32:111-6.

${ }^{19}$ Hoyt CS, Billson FA. Optic neuropathy in ketogenic diet. $\mathrm{Br} \mathrm{J}$ Ophthalmol 1979;63:191-4.

${ }^{20}$ Sills M, Forsythe WI, Haidukewych D. Role of octanoic and decanoic acids in the control of seizures. Arch Dis Child 1986;61:1173-7.

Correspondence to $\mathrm{Dr}$ W I Forsythe, Clarendon Wing, The General Infirmary at Leeds, Belmont Grove, Leeds LS2 9NS, England.

Received 7 July 1986

\title{
Twenty five years ago
}

\section{Disorders of spoken language in young children}

\author{
Mary D Sheridan (London)_Arch Dis Child 1961;36:11-16
}

'The normal acquisition in early childhood of all the biologically determined skills depends upon timely opportunity to explore, experiment with and learn to understand the world about him, and to realize how he relates to other people. If, through congenital disability or environmental deprivation, the normal methods of learning and communication are not available to him, he must substitute whatever means are open to him, or fail to develop his personality and his intellect. This biological learning appears to reach its zenith in the first two or three years of life and gradually tapers off by the age of 6 or 7 years, after which habits of functioning appear to be firmly fixed.'

Dr Sheridan classifies and discusses the reasons for delay or failure in the development of spoken language under the following headings: impaired hearing; low intelligence; delayed maturation of the central nervous system; brain injury; psychogenic disorders; motor dysfunctions; lesions of the peripheral speech organs.

'The commonest causes of delay in the development of spoken language are deafness and mental backwardness. Regarding the rarer causes of delay it needs to be remembered that lesions of the central nervous system occurring in the first few years of life have been inflicted on plastic and rapidly growing tissues, and only time can tell whether the resulting damage is permanent, or capable of compensation, or open to recovery. The first five or six years are therefore critical for diagnosis, treatment and training.'

(Those who were privileged to know Mary Sheridan quickly realised that they were in the presence of an exceptional person, in spite of her oft repeated protestations that she was only a simple school medical officer reluctantly presenting her findings to the experts. She was a true pioneer and her methods of assessment, particularly in the auditory and visual fields, have stood the test of time. There can be few people whose researches have contributed more to the assessment and care of the handicapped child .... . NEIL GORDON.) 